\title{
Accesibilidad de información en la web de instituciones oficiales de Paraguay
}

Information accessibility of official institutions of Paraguay websites

\author{
Maria Felicia Chamorro \\ Universidad Americana, Paraguay \\ mariafelichamorro@gmail.com
}

(D) https://orcid.org/0000-0002-3781-0344

\author{
Alicia Duarte Caballero \\ Universidad Americana, Paraguay \\ aliciaduarte09@gmail.com \\ (D) https://orcid.org/0000-0002-4750-0737
}

\author{
Nelly Manuela Calderón Giménez \\ Universidad Americana, Paraguay \\ nellycalderon3@gmail.com \\ (DD https://orcid.org/0000-0001-7945-0482
}

\author{
Sergio Duarte Masi \\ Universidad Americana, Paraguay \\ seduma@gmail.com \\ (D) https://orcid.org/0000-0002-9771-3888
}

\author{
Viviana Elizabeth Jiménez Chávez \\ Universidad Americana, Paraguay \\ vmonges0212@gmail.com \\ (DD https://orcid.org/0000-0002-9442-5039
}

\section{Resumen:}

El acceso a la información está plenamente garantizado por las legislaciones nacionales e internacionales. Las instituciones, principalmente públicas, deben asegurarse de que sus portales de internet sean utilizables por las personas independientemente de su condición. Este trabajo verificó el cumplimiento de las pautas de la WCAG 2.0 de las páginas oficiales de los Ministerios de Paraguay, utilizando la herramienta de evaluación TAW disponible en línea. Los resultados muestran ausencia de los estándares de accesibilidad. Las entidades del estado paraguayo deberían considerar la importancia de crear sitios web accesibles a fin de contribuir con la inclusión social de sectores de la población, principalmente personas con discapacidad y adultos mayores.

Palabras clave: Accesibilidad web, Acceso a información, Información pública, Personas con discapacidad, Paraguay.

\begin{abstract}
:
Access to information is fully guaranteed by national and international legislation. Institutions, mainly public, must guarantee that their internet portals are usable by people regardless of their condition. This work verified compliance with the WCAG 2.0 guidelines of the official pages of the Ministries of Paraguay, using the TAW evaluation tool available online. The results show the absence of accessibility standards. Paraguayan state entities should consider the importance of creating accessible websites in order to contribute to the social inclusion of sectors of the population, mainly people with disabilities and the elderly.
\end{abstract}

KeYwords: Web accessibility, Information Access, Public Information, Persons with Disabilities, Paraguay. 


\section{INTRODUCCIÓN}

Contar con sitios web accesibles a su información constituye un elemento clave de inclusión social y un derecho humano fundamental. Resulta sabido que buscar, recibir y difundir información está plenamente garantizado por la legislación tanto en el territorio nacional como internacional (Ley 5.282, 2014; ONU, 2006; ORSI, 2008, Reisdorf y Rhinesmith, 2020).

Por su parte, las Tecnologías de la Información y la Comunicación (TIC), se han insertado en la sociedad produciendo cambios en diversos aspectos de la vida de las personas. Es un elemento que permite ejercer el derecho a la educación, al trabajo, a la salud y a servicios e informaciones (ORSI, 2008; Reisdorf y Rhinesmith, 2020).

En este sentido, la accesibilidad web (AW) cobra vital importancia para favorecer la inclusión efectiva. En concreto, lo que se pretende es que un sitio web pueda ser utilizada por todas las personas en forma favorable en igualdad de condiciones (Acevedo, Gómez Solis, Mariño, y Godoy, 2013; SGAD, 2019).

De acuerdo con Arrufat Pérez de Zafra y Alcain Martínez (2018, p. 29), la AW es el "conjunto de principios y técnicas que se deben respetar al diseñar, construir, mantener y actualizar los sitios web y las aplicaciones para dispositivos móviles de modo a garantizar la igualdad y la no discriminación”. Por ende, el incumplimiento de los estándares supone una barrera a la que deben enfrentarse determinados grupos de usuarios cuando pretenden utilizar las TIC, ocasionándoles exclusión y hasta discriminación (Ferreira y Díaz Velázquez, 2009; Reisdorf y Rhinesmith, 2020).

Mejorar el acceso a los contenidos web constituye una preocupación de varios organismos que promueven la accesibilidad universal, los cuales llevaron adelante estudios sobre la temática. En tal sentido, el Observatorio Regional de la Sociedad de la Información dependiente de la Junta de Castilla y León realizó una de las primeras investigaciones, destacando a las herramientas TIC que ayudan a las personas con discapacidad para usar internet (ORSI, 2008). Asimismo, el Observatorio de Infoaccesibilidad de Discapnet (2008), fue uno de los primeros en realizar estudios sobre portales de los ministerios en España, en el que se analizaron 17 de ellos.

La iniciativa World Wide Web Consortium (W3C), organización internacional que trabaja para desarrollar estándares web que permitan contar con portales accesibles estableció las primeras pautas en el año 1999, la WCAG 1.0, que fue la primera guía para programadores (Fontanet Nadal, y Jaurne Mayol, 2011). Posteriormente, esta iniciativa publicó la segunda versión conocida por su sigla en inglés por WCAG 2.0 de la W3C. La guía ofrece cuatro principios básicos fundamentales: perceptibilidad, operabilidad, comprensibilidad y robustez (Sam Anlas y Stable Rodríguez, 2015; W3C, 2019). Los cuales, además, albergan doce pautas generales que establecen lo esencial para crear un sitio web accesible.

Para conocer el nivel de accesibilidad de un sitio web, existen diversas herramientas de validación que ayudan a comprobar el cumplimiento de los estándares (López-Zambrano, Moreira-Pico y Alava-Cagua, 2018; Mariño y Alfonzo, 2017; Paniagua, Bedoya, y Mera, 2020; Serrano Mascaraque, 2009; SGAD, 2019). Una de ellas es TAW (Test de Accesibilidad Web), la cual permite analizar en forma automática las pautas de la WCAG en línea y sin costo. TAW ofrece tres niveles de análisis:

- A: cumple todos los puntos de verificación de prioridad 1.

- AA: cumple todos los puntos de verificación de prioridad 1 y 2

- AAA: cumple todos los puntos de verificación de prioridad 1,2 y 3.

A su vez, el análisis se divide en dos categorías:

- Automáticos: problemas de accesibilidad que la herramienta detecta por si sola y que deben ser solucionados. 
- Manuales: la herramienta señala la existencia de un posible problema que el evaluador/a debe confirmar o descartar.

Los resultados se presentan en tres aspectos: problemas, advertencias y los elementos no verificados que requieren de una verificación manual (Riaño Herrera y Ballesteros Ricaurte, 2014; Sam Anlas y Stable Rodríguez, 2015; TAW, 2019).

En Paraguay, según la Ley 5.282/14, todo ciudadano sin discriminación alguna debe tener garantizado el uso de la información pública, en forma gratuita y sin necesidad de justificar las razones por la que formula su pedido (Ley 5.282, 2014). Si bien el país no cuenta con una ley exclusiva de accesibilidad web, además del marco normativo ya mencionado, cuenta con una Política Pública de Acción Nacional por los Derechos de las Personas con Discapacidad, que contiene las acciones a realizar en el quinquenio 2015 al 2030, para el logro efectivo de los derechos de las personas con discapacidad. Entre otros lineamientos, incluye la garantía de la accesibilidad efectiva a los servicios de comunicación, a la información/comunicación, incluyendo las TIC (SENADIS, 2015). Igualmente, en el decreto reglamentario de la Ley de Acceso a Información Pública, en el artículo 7, está claramente establecido que las fuentes públicas deben "...incorporar gradualmente soluciones tecnológicas que eliminen o disminuyan los obstáculos para las personas con discapacidad" (Paraguay. Ministerio de Justicia, 2015). Sin embargo, en los lineamientos y guías técnicas nacionales para la creación de sitios web no se contempla la implementación de estándares de accesibilidad web (SENATICs, 2015; 2016).

Por tanto, el estado debe asegurar condiciones de igualdad para el acceso a la información pública, a fin de garantizar los derechos de las personas con discapacidad. En este sentido, el presente trabajo define como objetivo verificar el cumplimiento de las pautas de la WCAG 2.0 en las páginas oficiales de los Ministerios de Paraguay, utilizando la herramienta de evaluación TAW disponible en línea.

\section{Metodología}

La metodología planteada en este estudio fue de diseño descriptivo, de corte transversal con enfoque cuantitativo. La muestra analizada constituyó los sitios web de 18 ministerios del estado paraguayo. El análisis fue aplicado en el mes de septiembre del año 2019, mediante el Test de Accesibilidad Web en línea (CTIC, 2019).

Los datos fueron recopilados en una planilla Excel para su análisis y presentados en una tabla y figuras según los niveles de conformidad (A, AA y AAA), distribuido en categorías proveídos por el validador TAW: "Problemas, Advertencias y No verificados". Se omiten los nombres de las instituciones ya que este estudio solo busca describir las situaciones en las que se encuentran los espacios de acceso a la información pública en internet, de modo que los tomadores de decisiones puedan tener en cuenta las medidas pertinentes para asegurar el acceso en igualdad de condiciones.

\section{Resultados y Discusión}

En la Tabla 1 se muestran los resultados de la evaluación de accesibilidad web generados por el validador TAW que detectó los problemas, las advertencias y los elementos no verificados que requieren de una verificación manual para los tres niveles de conformidad A, AA, AAA. 
TABLA 1

Resultados de análisis en los niveles A, AA y AAA.

\begin{tabular}{|c|c|c|c|c|c|c|c|c|c|}
\hline \multirow[t]{2}{*}{ Portales } & Problemas & Advertencias & \begin{tabular}{|l} 
No \\
Verificado
\end{tabular} & Problemas & Advertencias & \begin{tabular}{|l} 
No \\
Verificado
\end{tabular} & Problemas & Advertencias & \begin{tabular}{|l} 
No \\
Verificado
\end{tabular} \\
\hline & \multicolumn{3}{|c|}{ A } & \multicolumn{3}{|c|}{ AA } & \multicolumn{3}{|l|}{$\mathrm{AAA}$} \\
\hline Sitio 1 & 7 & 2 & 11 & 7 & 2 & 17 & 12 & 2 & 28 \\
\hline Sitio 2 & 43 & 164 & 10 & 43 & 184 & 164 & 47 & 198 & 27 \\
\hline Sitio 3 & 56 & 58 & 8 & 56 & 93 & 14 & 65 & 128 & 25 \\
\hline Sitio 4 & 4 & 2 & 12 & 43 & 106 & 14 & 50 & 101 & 25 \\
\hline Sitio 5 & 86 & 688 & 10 & 86 & 760 & 16 & 89 & 792 & 27 \\
\hline Sitio 6 & 32 & 134 & 11 & 32 & 207 & 17 & 36 & 212 & 28 \\
\hline Sitio 7 & 74 & 695 & 10 & 74 & 779 & 16 & 85 & 826 & 27 \\
\hline Sitio 8 & 43 & 205 & 25 & 42 & 200 & 14 & 43 & 200 & 25 \\
\hline Sitio 9 & 6 & 2 & 12 & 38 & 374 & 12 & 6 & 1 & 29 \\
\hline Sitio 10 & 42 & 85 & 9 & 42 & 145 & 15 & 54 & 198 & 26 \\
\hline Sitio 11 & 97 & 107 & 8 & 97 & 184 & 14 & 126 & 263 & 25 \\
\hline Sitio 12 & 44 & 4 & 9 & 44 & 177 & 15 & 57 & 234 & 26 \\
\hline Sitio 13 & 36 & 57 & 8 & 36 & 110 & 14 & 57 & 146 & 25 \\
\hline Sitio 14 & 22 & 229 & 9 & 22 & 253 & 15 & 30 & 277 & 26 \\
\hline Sitio 15 & 29 & 167 & 8 & 29 & 196 & 14 & 31 & 242 & 25 \\
\hline Sitio 16 & 136 & 148 & 11 & 36 & 197 & 17 & 48 & 394 & 28 \\
\hline Sitio 17 & 87 & 154 & 9 & 87 & 279 & 14 & 92 & 343 & 25 \\
\hline Sitio 18 & 37 & 65 & 10 & 39 & 84 & 16 & 69 & 103 & 27 \\
\hline
\end{tabular}

Fuente: elaboración propia.

Como puede notarse en la Tabla 1, de acuerdo con el validador utilizado, los resultados indican que los sitios analizados no cumplen con los criterios básicos relacionados a los niveles de conformidad establecidos por la WCAG para permitir que ciertos grupos de usuarios utilicen la web. Por tanto, se puede considerar que los portales de los ministerios estudiados no utilizan las recomendaciones de accesibilidad a fin de considerarse como un sitio accesible.

$\mathrm{Al}$ respecto, investigaciones previas relacionadas a la evaluación de los niveles de accesibilidad teniendo en cuenta las pautas WCAG en los portales oficiales de autores tales como Ortiz Ruiz (2019) y Sam Anlas y Stable Rodríguez (2016), que han llevado a cabo un análisis para evaluar la accesibilidad de los portales de los ministerios de Chile y Perú respectivamente, han concluido que los portales analizados presentan barreras para acceder a la información contenida en estos sitios oficiales, coincidiendo los resultados que se presentan en este estudio. En el caso chileno, se examinaron los sitios web informativos y de consulta desarrollados por el Ministerio de Educación de Chile, según las recomendaciones del Servicio Nacional de la Discapacidad (SENADIS) de Chile, mientras que la investigación referente al Ministerio del Perú seleccionó los portales más citados en el proyecto de gobierno electrónico.

Se considera oportuno mencionar que esta situación podría subsanarse sencillamente al tener presente a los sectores minoritarios a la hora del diseño y desarrollo de productos web, principalmente en los entes públicos, dado que está demostrado que tener un sitio accesible puede lograrse al instalar el tema como una buena práctica para incorporar las pautas de accesibilidad de acuerdo a las recomendaciones de la W3C, lo cual no implica una inversión económica adicional (Barros, Campo y Galaz, 2015; Mariño y Alfonzo, 2017).

El nivel A representa el nivel mínimo de adecuación que debe cumplir un sitio web para que pueda accederse a su contenido. En la figura 1 se muestra que para el nivel de análisis A, la puntuación 136 representa el mayor número de problemas de accesibilidad detectado, que corresponde al Sitio 16. El promedio de problemas que se encuentra en este nivel de análisis es de 50. Las advertencias, que deben revisarse manualmente, puntúan un total de 695, que corresponde al Sitio 7. 
FIGURA 1

Nivel de análisis A: total de problemas detectados, advertencias y elementos no verificados.

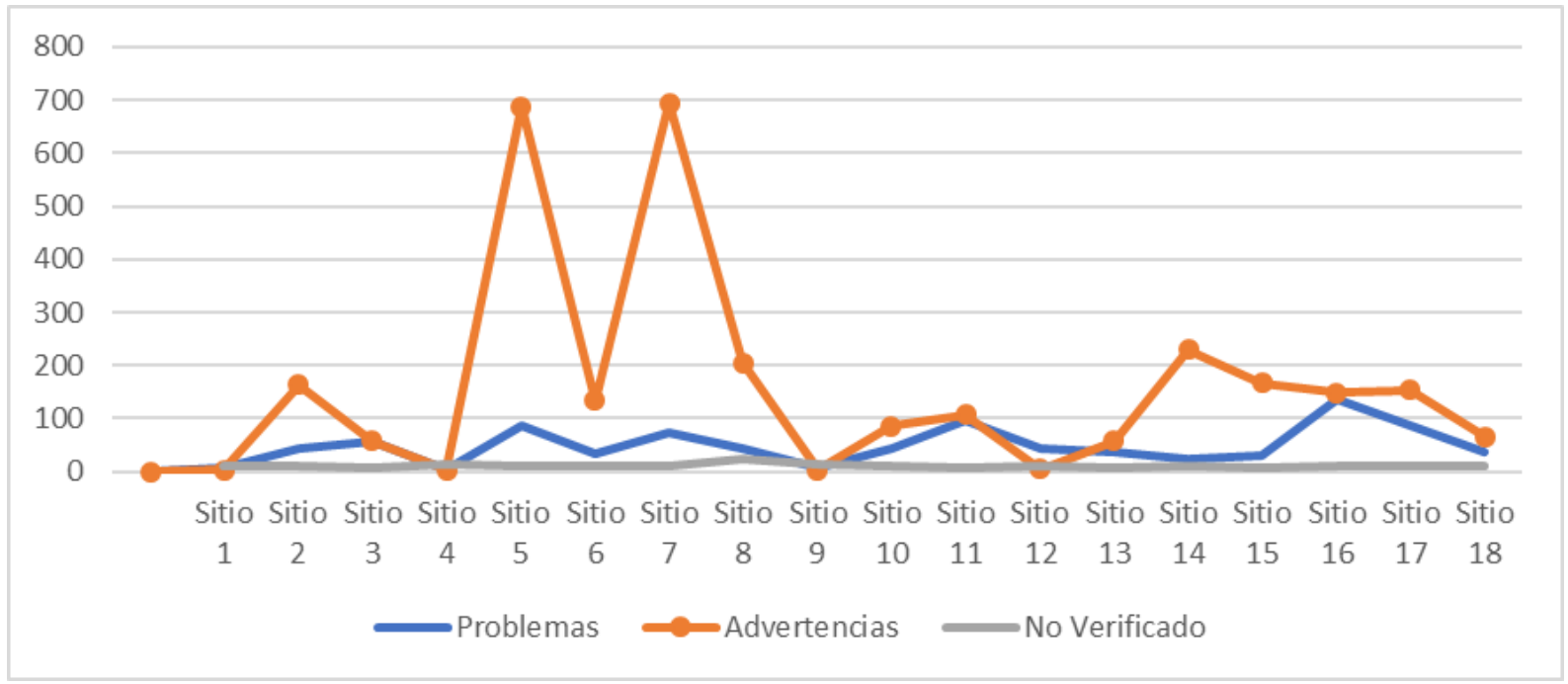

Fuente: elaboración propia.

El hecho de no satisfacerse este nivel, implica que un grupo de personas serán incapaces de acceder a la información del sitio. Facilitar el acceso a la información es un compromiso de las instituciones del estado, que deben tomar conciencia y trabajar en el desarrollo de plataformas tecnológicas accesibles; dando cumplimiento a lo establecido en la Convención por los Derechos de las Personas con Discapacidad y el Plan de Acción Nacional por los Derechos de las Personas con Discapacidad 2015-2030 (SENADIS, 2015).

La figura 2 muestra el nivel de análisis AA, este criterio de conformidad corresponde a un nivel intermedio de cumplimiento de las pautas para considerar a un sitio accesible. Implica el cumplimiento de los criterios requeridos en los niveles A y AA. La evaluación automática a este nivel encontró hasta 97 problemas que corresponden al Sitio 11 y 779 advertencias que corresponde al Sitio 7.

\section{FIGURA 2}

Nivel de análisis AA: total de problemas detectados, advertencias y elementos no verificados.

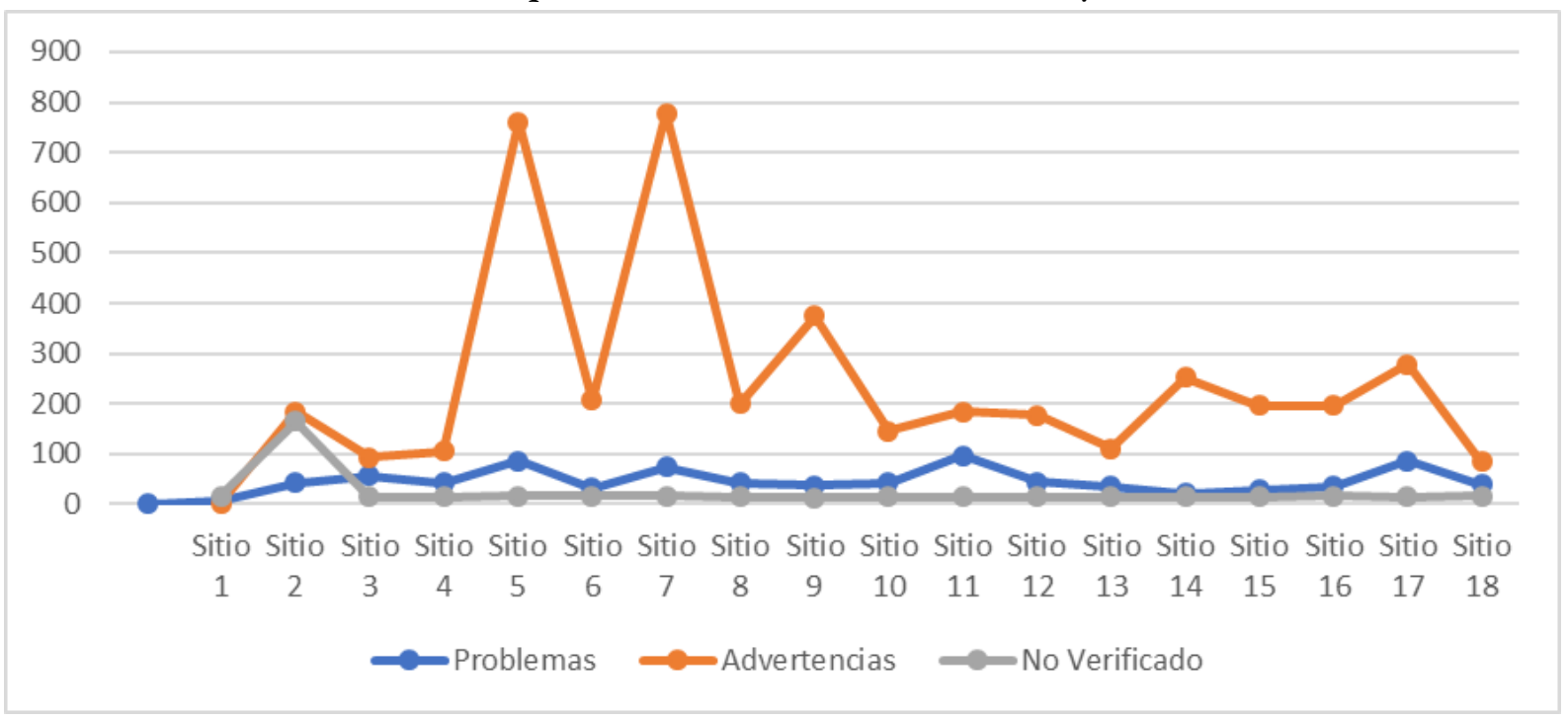

Fuente: elaboración propia. 
La ausencia de cumplimiento de este nivel confirma que un grupo de usuarios está pasando por muchas dificultades para lograr obtener acceso al contenido web que proporciona la institución, en nuestro caso, los entes públicos.

En el nivel de análisis AAA (Figura 3), es el nivel máximo de accesibilidad. Una web que obtenga el nivel AAA cumple los criterios establecidos en los niveles A, AA, AAA y es considerado accesible para todos los usuarios. En este nivel de adecuación los resultados indican que 126 es el número mayor de problemas detectados que corresponde al Sitio 11. Las advertencias hasta 826 que corresponden al Sitio 7.

FIGURA 3

Nivel de análisis AAA: total de problemas detectados, advertencias y elementos no verificados.

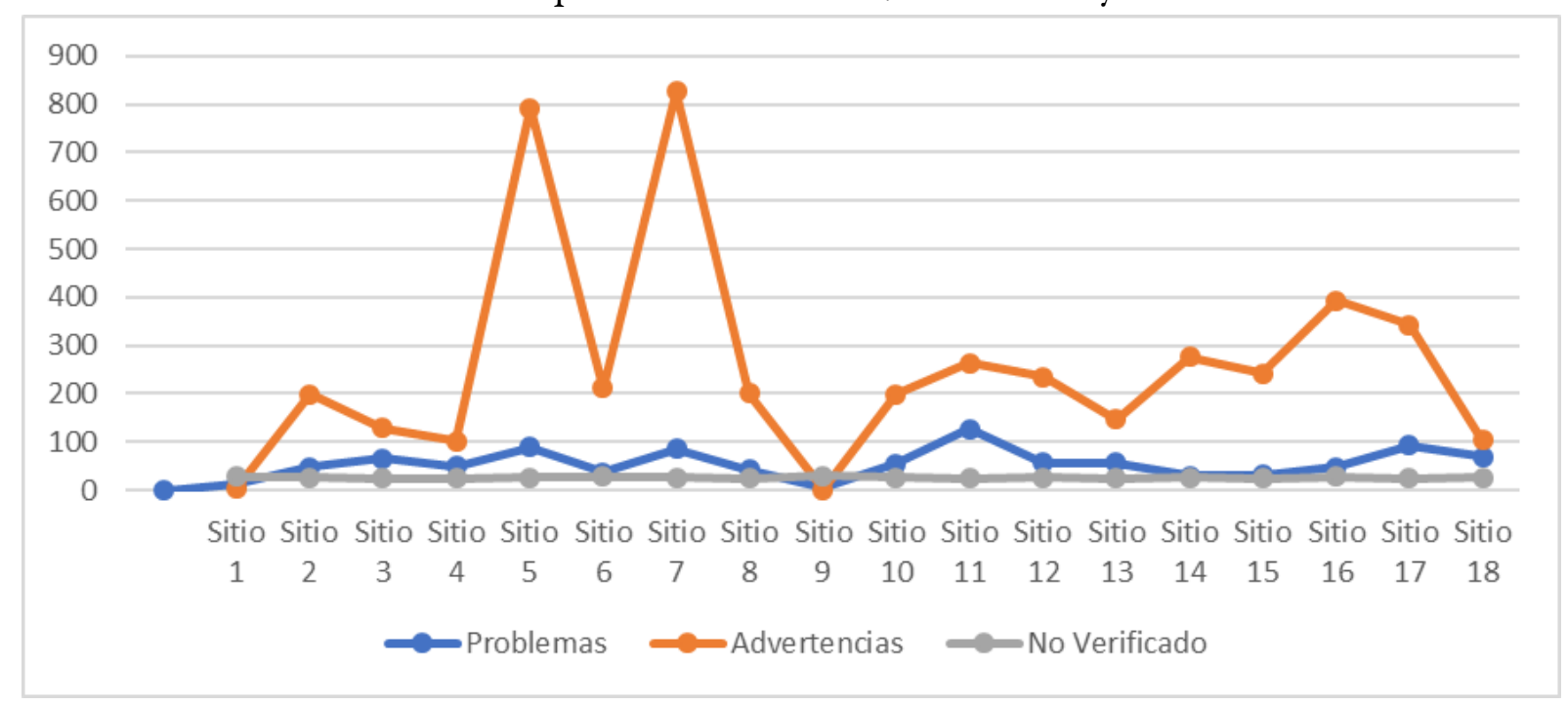

Fuente: elaboración propia.

No cumplir con este nivel significa que para acceder a la información algunas personas se encontraran con dificultades. Es importante puntualizar que el cumplimiento de las normas de accesibilidad permite la utilización del mayor número de personas, incluidas los adultos mayores que presentan dificultades relacionados con el envejecimiento tal como lo señala Fuertes y Martínez (2012).

\section{Conclusiones}

Los resultados proporcionados por el validador TAW, con referencia a los niveles de accesibilidad de las páginas de internet analizados, indican que las páginas no alcanzan el nivel mínimo, lo que impide su utilización en igualdad de condiciones con los demás usuarios.

En este sentido, es importante tener en cuenta que las recomendaciones del consorcio W3C son una referencia para seguir y las instituciones públicas deben considerar su implementación, a fin de contribuir a la inclusión social de sectores de la población que tienen dificultades para acceder a los contenidos web proporcionados por el estado.

Si bien para que el estudio sea más completo se recomienda la verificación manual de los "No verificados", el cual no se contempló en este trabajo, consideramos que es sumamente transcendental los resultados para que se pueda tener presente el tema de la accesibilidad en los lineamientos y guías técnicas proporcionados por el estado con relación a la elaboración de productos de información a ser proveía para la ciudadanía mediante la web. 


\section{REFERENCIAS}

Acevedo, J., Gómez Solis, L., Mariño, S. y Godoy, M. (2013). Guidelines for evaluating web accessibility, level A. Journal of computer science and technology, 13(2), 76-83. Recuperado de https://pdfs.semanticscholar.org/93ed/b31f0f178accfc580b91b3d489c03da238f1.pdf?_ga=2.13303732.2 25154378.1596903062-1553294703.1592498890

Arrufat Pérez de Zafra, M. A. y Alcain Martínez, E. (2018). La accesibilidad de los sitios web y aplicaciones para dispositivos móviles del sector público. Madrid: CERMI. Recuperado de https://www.cermi.es/sites/default/files /docs/colecciones/coleccion\%20Inclusi\%C3\%B3n\%20y\%20diversidad\%20n\%C2\%BA26_ONU.pdf

Barros, A., Campo, R. y Galaz, P. (2015). La accesibilidad, una obligación de los Estados en sus servicios web. Series sistemas públicos, 12, 1-22. Recuperado de https://www.alejandrobarros.com/wp-content/uploads/2016/04/A ccesibilidad-Sistemas-Pu\%CC\%81blicos.pdf

CTIC. (2019). TAW Test de accesibilidad web. Recuperado de https://www.tawdis.net/?lang=es

Ferreira, M. A. V. y Díaz Velázquez, E. (2009). Discapacidad, exclusión social y tecnologías de la información. Política y sociedad, 46(1y2), 237-253. Recuperado de https://www.researchgate.net/publication/277258896_Discapac idad_exclusion_social_y_tecnologias_de_la_informacion

Fontanet Nadal, G. y Jaurne Mayol, J. (2011). Importancia y situación actual de la accesibilidad web para el turismo accesible. Pasos: revista de turismo y patrimonio cultural, 9(2), 317-326. Recuperado de https://riull.ull.es/xmlu i/bitstream/handle/915/16012/PS_9_2\%20_\%282011\%29_08.pdf?sequence=1\&isAllowed =y

Fuertes, J. y Martínez, L. (2012). Accesibilidad a la web: legislación y estándares. Recuperado de https://core.ac.uk/d ownload/pdf/61912290.pdf

Ley 5.282 (2014). Ley de libre acceso ciudadano a la información pública y transparencia gubernamental. Asunción, Paraguay. Recuperado de http://www.senadis.gov.py/archivos/documentos/ley\%205282_1wzzzukq.pdf

López-Zambrano, J., Moreira-Pico, J. y Alava-Cagua, N. (2018). Metodología para valorar y clasificar herramientas de evaluación de accesibilidad web. e-Ciencias de la información, 8(1), 172-189. Recuperado de https://revistas.uc r.ac.cr/index.php/eciencias/article/view/30012

Mariño, S. y Alfonzo, P. (2017). Evaluación de la accesibilidad web: una mirada para asegurar la formación en la temática. Campus virtuales, 6(2), 21-30. Recuperado de http://uajournals.com/ojs/index.php/campusvirtuales /article/view/216/197

Observatorio de la Infoaccesibilidad de Discapnet. (2008). Accesibilidad en losportales de los ministerios. Recuperado de https://www.discapnet.es/sites/default/files/areas-tematicas/tecnologia/informe_detallado_ministerios.pdf

ONU. (2006). Convención sobre los derechos de las personas con discapacidad. Recuperado de https://www.ohchr.org/ Documents/Publications/AdvocacyTool_sp.pdf

ORSI (Observatorio Regional de Información). (2008). E-accesibilidad: eliminación de barreras para el acceso a la sociedad digital del conocimiento. Recuperado de http://bibliotecadigital.jcyl.es/es/consulta/registro.cmd?id=1 8578

Ortiz Ruiz, Y. (2019). Accesibilidad en sitios web del Ministerio de Educación de Chile. Tend pedagó, (33), 99-116. Recuperado de https://repositorio.uam.es/handle/10486/686427

Paniagua, A., Bedoya, D., Mera, C. (2020). Un método para la evaluación de la accesibilidad y la usabilidad en aplicaciones móviles. TecnoLógicas, 23(48). Recuperado de http://www.redalyc.org/articulo.oa?id=344263272 016

Paraguay. Ministerio de Justicia. (2015). Decreto $N^{o}$ 4064. Que reglamenta la Ley $N^{o} 5282$ de libre acceso ciudadano a la información pública y transparencia gubernamental. Asunción, Paraguay. Recuperado de https://www.mitic.gov.py/application/files/4115/4877/9341/DECRETO_4064_15_Reglamento_Ley_d e_Acceso_a_la_Informacion_5282-2014.pdf

Reisdorf, B. y Rhinesmith, C. (2020). Digital inclusion as a Core Component of social inclusion. Social inclusion, 8(2), 132-137. Recuperado de https://www.cogitatiopress.com/socialinclusion/article/viewFile/3184/3184 
Riaño Herrera, J. y Ballesteros Ricaurte, J. (2014). Aspectos y normas de accesibilidad web. Ing USBMed, 5(2), 26-32. Recuperado de http://revistas.usbbog.edu.co/index.php/IngUSBmed/article/view/308/219

Sam Anlas, A. y Stable Rodríguez, Y. (2015). Evaluación de la accesibilidad web del portal de la biblioteca nacional del Perú. Bibliotecas. Anales de Investigación, 11(11), 224-231. Recuperado de https://dialnet.unirioja.es/servle t/articulo? codigo $=5704531$

Sam Anlas, C. y Stable Rodríguez, Y. (2016). Evaluación de la accesibilidad web de los portales del Estado en Perú. Revista española de documentación cientifica, 39(1), e120. Recuperado de http://redc.revistas.csic.es/index.php $/$ redc/article/view/923/1337

SENADIS (Secretaria Nacional por los Derechos de las Personas con Discapacidad). (2015). Plan de Acción Nacional por los Derechos de las Personas con Discapacidad 2015-2030. Fernando de la Mora, Paraguay: SENADIS. Recuperado de https://acnudh.org/load/2019/07/047-Plan-de-Acci\%C3\%B3n-Nacional-DPcD.pdf

SENATICs. (2015). Guia 1: Normativas técnicas para sitios web acceso a la información pública. Asunción, Paraguay: SENATICs. Recuperado de https://gestordocumental.mitic.gov.py/share/s/L3iLMLyLQ4Cv7W4HD_LCd A

SENATICs. (2016). Manual de uso Portal de solicitudes de información pública: Perfil funcionario encargado de la oficina de acceso a la información. Asunción, Paraguay: SENATICs. Recuperado de https://gestordocumental.mitic.go v.py/share/s/BN8bauR7TvKTXdGkGqyc3Q

Serrano Mascaraque, E. (2009). Herramientas para la evaluación de la accesibilidad web. Documentación de las ciencias de la información, 32, 245-266. Recuperado de https://revistas.ucm.es/index.php/DCIN/article/download/D CIN0909110245A/18823/

SGAD (Secretaría General de Administración Digital). (2019). Guia de validación de accesibilidad web (2 ed.). Recuperado de https://administracionelectronica.gob.es/pae_Home

W3C. (2019). Web Accessibility Initiative WAI. Recuperado de https://www.w3.org/WAI/ 